\title{
Síntesis, caracterización y evaluación eléctrica de circonatos de bario dopados con lantánidos trivalentes
}

\author{
O. A. GERENA ${ }^{1 *}$, J. B. CARDA ${ }^{2}$, H. BELTRÁN ${ }^{2}$, E. CORDONCILLO ${ }^{2}$ Y J. S. VALENCIA ${ }^{1}$ \\ 'Laboratorio de Catálisis Heterogénea, Departamento de Química, Facultad de Ciencias, Universidad Nacional de Colombia, \\ Bogotá, D.C., Colombia \\ 22Departamento de Química Inorgánica y Orgánica, Universitat Jaume I de Castelló, Castellón de la Plana, España \\ *e-mail: oagerenar@unal.edu.co
}

\begin{abstract}
El circonato de bario es un material oxídico con estructura de tipo perovskita que muestra alta estabilidad química tanto en ambientes oxidantes como reductores, como en presencia de agua y dióxido de carbono; su conductividad ha permitido examinarlo como electrolito para celdas de combustible de óxido sólido encontrando buenos resultados, con la limitante de operar a temperaturas superiores a $\operatorname{los} 800^{\circ} \mathrm{C}$.

Diversos investigadores han propuesto que es posible mejorar su conductividad eléctrica por modificaciones en su composición química, en particular por dopaje con cationes trivalentes que reemplacen el circonio en el sitio B de la perovskita. En este estudio se sintetizó el circonato de bario por el método citrato amorfo a fin examinar la posibilidad de obtenerlo en condiciones más favorables que las presentadas por el método de síntesis convencional (método cerámico o reacción de estado sólido). Se preparó circonato de bario dopado con europio, gadolinio, holmio, lantano, neodimio y praseodimio; la identificación de fases presentes se verificó por difracción de rayos X (DRX), las propiedades eléctricas se examinaron por espectroscopía de impedancias (IS) a temperaturas entre 480 y $680^{\circ} \mathrm{C}$, con miras a evaluar su potencial uso como electrolito en celdas de combustible de óxido sólido.

Los aportes de esta investigación se han centrado en el método de síntesis; en la obtención de polvos cerámicos de circonato de bario a temperaturas inferiores a las requeridas por el método cerámico; en la obtención de información química, estructural, morfológica y eléctrica de los materiales sintetizados.

Se encontró la fase deseada en las condiciones de síntesis establecidas, así mismo, se aprecia un incremento significativo en la conductividad de los sólidos dopados con lantano, holmio y europio en relación al material sin dopaje alguno.
\end{abstract}

Palabras clave: circonato de bario, perovskita, conductor protónico, citrato amorfo.

Synthesis, characterization and electric evaluation of barium zirconate doped with trivalent lanthanides

Barium zirconate is an oxidic material having perovskite structure that exhibits high chemical stability in both oxidizing and reducing environments, such as in the presence of water and carbon dioxide, its conductivity has led to consider it as a electrolyte for solid oxide fuel cell finding good results, with the limitation of operating at temperatures above $800{ }^{\circ} \mathrm{C}$. Several researchers have proposed that it is possible to improve their electrical conductivity by changes in chemical composition, particularly for doping with trivalent cations that replace the zirconium in B site of perovskite. In this study, barium zirconate was synthetized by the amorphous citrate method to examine the possibility of obtaining in more favorable conditions than those made by the conventional method of synthesis (ceramic method or solid state reaction) conditions are synthesized.

Barium zirconate doped with europium, gadolinium, holmium, lanthanum, neodymium and praseodymium was prepared, the present phase identification was verified by X-ray diffraction (XRD), the electrical properties were examined by impedance spectroscopy (IS) at temperatures between 480 and $680^{\circ} \mathrm{C}$ in order to evaluate its potential use as a fuel cell electrolyte in solid oxide. The contributions of this research has focused on the synthesis method, in the production of ceramic powders of barium zirconate at temperatures lower than those required by the ceramic method, in obtaining chemical, structural, morphological and electrical information of material synthesized.

The desired phase synthesis conditions set found, also, a significant increase is seen in the solid conductivity of doped lanthanum, holmium and europium zirconate of barium in relation to the material without doping.

Keywords: Barium Zirconate, Perovskite, Protonic Conductor, Amorphous Citrate.

\section{INTRODUCCIÓN}

Factores como el alto precio de los combustibles fósiles, el interés por aumentar la eficiencia de su uso, la búsqueda de nuevas fuentes de energía, aunados al interés por la preservación del medio ambiente, son algunas de las razones que han suscitado un marcado interés por el estudio de dispositivos electroquímicos, como variantes válidas desde lo económico y lo ambiental, para la producción de energía. 
Las celdas de combustible han llamado la atención de científicos debido a sus altos valores de eficiencia y versatilidad en el uso de combustibles, en particular las celdas de combustible de óxido sólido (SOFC)(1). Sin embargo, estos dispositivos presentan inconvenientes ya que requieren temperaturas elevadas para su funcionamiento. Esta condición de operación ocasiona la rápida degradación de los componentes del dispositivo y el aumento de costo en su producción. Con el objeto de obtener celdas de combustible más rentables, los estudios se han dirigido a la búsqueda de materiales y condiciones que les permitan trabajar a temperaturas intermedias, en el intervalo comprendido entre 400 y $700{ }^{\circ} \mathrm{C}(2)$.

Operar en estas temperaturas ocasiona disminuciones significativas de la conductividad iónica del electrolito y de allí, una reducción en la eficiencia energética de las celdas de combustible, parámetro que las hace interesantes como sistema de generación de potencia. Para enfrentar este problema se han propuesto tres alternativas de mejora de las propiedades de los sólidos usados como electrolito(3). La primera alternativa se centra en su microestructura, en particular en el estudio de los efectos de los límites y el tamaño de grano a la par de las modificaciones de la estructura cristalina. La segunda alternativa se enfoca en las técnicas de procesamiento de los electrolitos, en especial en procesos de sinterización modificados a fin de obtener cerámicas con microestructura controlada (tamaño de grano, densidad, pureza). Por último, se busca mejorar la conductividad iónica de los electrolitos por medio de variaciones en la composición química.

En este sentido, se han estudiado materiales basados en óxidos de circonio, cerio, y mezclas de galio y lantano(4). Las modificaciones realizadas a estos materiales incluyen la adición de un dopante, generalmente óxidos de tierras raras. Una alternativa ha sido el estudio de sólidos que presenten una conductividad iónica dual, es decir, conduzcan iones óxido así como iones $\mathrm{H}^{+}$; en este sentido, algunas investigaciones han mostrado que óxidos con estructura tipo perovskita $\mathrm{ABO}_{3^{\prime}}$, como el cerato de bario $\left(\mathrm{BaCeO}_{3}\right)$, tantalato de potasio $\left(\mathrm{KTaO}_{3}\right)$ y el circonato de estroncio $\left(\mathrm{SrZrO}_{3}\right)$ se comportan como conductores protónicos(5) cuando en los sitios B de la perovskita se introducen dopantes catiónicos de menor número de oxidación. En este contexto se ha reportado que el cerato de estroncio $\left(\mathrm{SrCeO}_{3}\right)$ dopado con cationes de tierras raras (6-10) es un conductor protónico de alta temperatura. Este hecho ha generado un gran interés en estos materiales para posibles aplicaciones en las celdas de combustible, electrolitos de vapor, membranas para la separación de gases, células de hidrólisis, sensores de humedad y baterías de alta densidad.

En los sólidos estudiados se evidenció que la conductividad debida al movimiento de protones es muy inferior la conducción ocasionada por iones óxido (3) sin que por ello sea despreciable su aporte. Los resultados obtenidos sugieren que los óxidos compuestos basados en ceratos y circonatos de estroncio y bario $\left(\mathrm{SrCeO}_{3^{\prime}} \mathrm{BaCeO}_{3^{\prime}} \mathrm{BaZrO}_{3^{\prime}}, \mathrm{SrZrO}_{3}\right)$ presentan altos valores de conductividad protónica, lo cual ha permitido ensayarlos como electrolitos en celdas de combustible a escala de laboratorio(9)(10).

Los ceratos son los compuestos que mejores valores de conductividad exhiben pero, su estabilidad química en ambientes que incluyan agua es muy baja, por el contrario la estabilidad de los circonatos es mayor pero su conductividad es inferior. Como respuesta a estas limitaciones, se propone estudiar comportamiento del circonato de bario dopado con cationes de menor estado de oxidación, en particular lantánidos trivalentes(10).

Por sus especiales cualidades, el circonato de bario ha sido sintetizado usando una amplia gama de métodos que incluyen los de la vía seca como el método cerámico convencional y los de la química suave. Dentro de estos últimos, el método citrato amorfo(11), por su sencillez y muy fácil seguimiento, se usa en esta investigación. Esta ruta de síntesis permite calcinar las espumas precursoras a temperaturas inferiores a las requeridas por otros métodos de síntesis, reducir los tiempos de procesamiento, eliminar etapas intermedias de molienda y compresión de los materiales de síntesis. El método citrato amorfo se basa en la formación de especies complejas del ácido cítrico con los cationes metálicos, esto permite una muy buena dispersión de los materiales de partida en una disolución acuosa y conduce a la producción de espumas precursoras que luego de la calcinación rinden materiales policristalinos de tamaño nanométrico.

En esta investigación se preparó por el método citrato amorfo circonato de bario, dopado con $0,3 \%$ de lantánidos trivalentes (europio, gadolinio, holmio, lantano, neodimio y praseodimio); se verificó la obtención de fases por análisis de difractometría de rayos $\mathrm{X}$ a 800,900 y $1000{ }^{\circ} \mathrm{C}$ y se evaluaron las propiedades eléctricas del circonato de bario dopado y sin dopar por espectroscopía de impedancias a fin de explorar su posible uso como electrolito en celdas de combustible de óxido sólido.

\section{PARTE EXPERIMENTAL}

Para la preparación de los sólidos se utilizaron oxicloruro de circonio (SIGMA-ALDRICH), nitrato de bario (SIGMAALDRICH), ácido cítrico (MERCK), amoniaco $27 \%$ (MERCK), óxidos de europio, gadolinio, holmio, lantano y neodimio (METALL RARE EARTH); isopropóxido de praseodimio (MERCK), sin posteriores tratamientos.

La síntesis de los precursores se verificó por el método de química suave denominado citrato amorfo; para ello se prepararon disoluciones acuosas de ácido cítrico, oxicloruro de circonio, nitratos de bario y de los lantánidos trivalentes por acción de ácido nítrico sobre el óxido respectivo; como fuente de praseodimio se usó su isopropóxido atacado por una disolución de ácido nítrico.

Al volumen adecuado de la disolución de ácido cítrico se adicionaron con agitación constante y en estricto orden, los volúmenes requeridos de disoluciones de nitrato de bario, oxicloruro de circonio y del lantánido trivalente. Se ajustó el $\mathrm{pH}$ a 3,50 por adición de amoniaco acuoso; se llevaron a un mismo volumen todas las mezclas preparadas, que fueron luego sometidas a reflujo por 4 horas.

Luego se evaporó el agua a presión atmosférica y el material así obtenido se secó posteriormente a $100{ }^{\circ} \mathrm{C}$ por 10 horas, encontrando, de esta forma las espumas precursoras que, en tratamiento posterior, se calcinaron en atmósfera de aire.

La rutina para el tratamiento térmico de las espumas precursoras inicia a temperatura ambiente y se calienta a $10{ }^{\circ} \mathrm{C}$ por minuto hasta $800^{\circ} \mathrm{C}$, se mantiene a esta temperatura por 60 minutos y por último a $1000{ }^{\circ} \mathrm{C}$ por 30 minutos.

En la Tabla 1 se registran las cantidades de reactivos usados para los ensayos y la nomenclatura usada.

La identificación de las fases presentes en los sólidos preparados se realizó por difractómetría de rayos $X$ en un difractómetro PANalytical modelo X’Pert Pro MPD, equipado 
TABla 1. ParÁMETROS PARA LA Síntesis de CiRCONATO DE BARIO DOPADO.

\begin{tabular}{|c|c|c|c|c|c|}
\hline \multirow{2}{*}{ Compuesto } & \multicolumn{5}{|c|}{ Cantidad adicionada (mmol) } \\
\cline { 2 - 6 } & $\mathbf{B a}$ & $\mathbf{Z r}$ & $\mathbf{L n}$ & H $_{3}$ Cit & 20 \\
\hline $\mathrm{BaZrO}_{3}$ & 10 & 10 & 0 & 20 & $\mathrm{~B}$ \\
\hline $\mathrm{BaZr}_{0,97} \mathrm{Eu}_{0,03} \mathrm{O}_{3-\delta}$ & 10 & 9,97 & 0,03 & 20 & $\mathrm{Eu}$ \\
\hline $\mathrm{BaZr}_{0,97} \mathrm{Gd}_{0,03} \mathrm{O}_{3-\delta}$ & 10 & 9,97 & 0,03 & 20 & $\mathrm{Gd}$ \\
\hline $\mathrm{BaZr}_{0,97} \mathrm{Ho}_{0,03} \mathrm{O}_{3-\delta}$ & 10 & 9,97 & 0,03 & 20 & $\mathrm{Ho}$ \\
\hline $\mathrm{BaZr}_{0,97} \mathrm{La}_{0,03} \mathrm{O}_{3-\delta}$ & 10 & 9,97 & 0,03 & 20 & $\mathrm{La}$ \\
\hline $\mathrm{BaZr}_{0,97} \mathrm{Nd}_{0,03} \mathrm{O}_{3-\delta}$ & 10 & 9,97 & 0,03 & 20 & $\mathrm{Nd}$ \\
\hline $\mathrm{BaZr0,97Pr0,03} \mathrm{O}-\delta$ & 10 & 9,97 & 0,03 & $\mathrm{Pr}$ \\
\hline
\end{tabular}

TABla 2. TAMAÑOS DE PARTíCULA Y ENERGÍAS DE ACTIVACIÓN (eV) PARA EL GRANO Y LÍMITE DE GRANO.

\begin{tabular}{|c|c|c|c|c|c|}
\hline \multirow{2}{*}{ Muestra } & \multirow{2}{*}{ Radio iónico dopante } & \multirow{2}{*}{$\mathrm{d}(\mathrm{nm})$} & \multicolumn{3}{|c|}{ Energías de activación } \\
\hline & & & Volumen de grano & Límite de grano & Total \\
\hline Blanco & - & 25 & 0,563 & 0,385 & 0,414 \\
\hline Ho & 0,901 & 35 & 0,685 & 0,658 & 0,662 \\
\hline $\mathrm{Gd}$ & 0,938 & 35 & 0,663 & 0,795 & 0,67 \\
\hline $\mathrm{Eu}$ & 0,947 & 48 & 0,524 & 0,725 & 0,713 \\
\hline $\mathrm{Nd}$ & 0,983 & 40 & - & - & - \\
\hline $\operatorname{Pr}$ & 0,99 & 43 & 0,845 & 0,819 & 0,837 \\
\hline $\mathrm{La}$ & 1,032 & 31 & 0,855 & 1,12 & 0,912 \\
\hline
\end{tabular}

con ánodo de cobre, usando la aplicación High Score Plus. Los parámetros para el análisis fueron: morfología de la muestra:

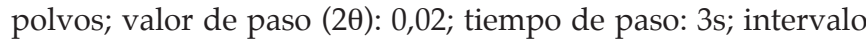
(20): $10^{\circ}-90^{\circ}$; temperatura ambiente.

Para el estudio de las propiedades eléctricas se dispuso la toma de mediciones de corriente alterna (AC), en un analizador de impedancias Agilent 4294A Impedance Analyser. A temperaturas comprendidas entre $500{ }^{\circ} \mathrm{C}$ y $700{ }^{\circ} \mathrm{C}$, haciendo un barrido de frecuencias entre 1 y $10^{7} \mathrm{~Hz}$, en atmósfera aire. Las muestras se prensaron a $2 \mathrm{Tn}$ en un troquel de $5 \mathrm{~mm}$ de diámetro con presión uniaxial, y se sinterizaron a $1000^{\circ} \mathrm{C}$ durante $6 \mathrm{~h}$. Todas las medidas están corregidas por el factor geométrico de cada pastilla analizada. Se permitió una estabilización de las muestras a la temperatura del análisis por 20 minutos antes de realizar las mediciones, se dispuso de electrodos de oro.

\section{RESULTADOS Y DISCUSIÓN}

\subsection{Identificación de fases}

El análisis por difracción de rayos $\mathrm{X}$ de los sólidos calcinados muestra que se obtiene la fase deseada, circonato de bario, mezclado con fases secundarias. La consulta con la base de datos del ICCD muestra concordancia con el compuesto de referencia $\mathrm{BaZrO}_{3^{\prime}}$ correspondiente al código de colección de la ICSD número 060399, con grupo espacial Pm-3m(221), y sistema cristalino cúbico. Usando la ecuación de Scherrer, se determinaron los tamaños de cristalito de las muestras analizadas; para tal efecto, se seleccionó la señal de mayor intensidad ubicada en $30,1^{\circ}$; en general, tal como se ve en la Tabla 2, los tamaños de partícula calculados de esta forma van desde $25 \mathrm{~nm}$ en el material sin dopar y entre 30 y $48 \mathrm{~nm}$ para los sólidos dopados. No es posible establecer

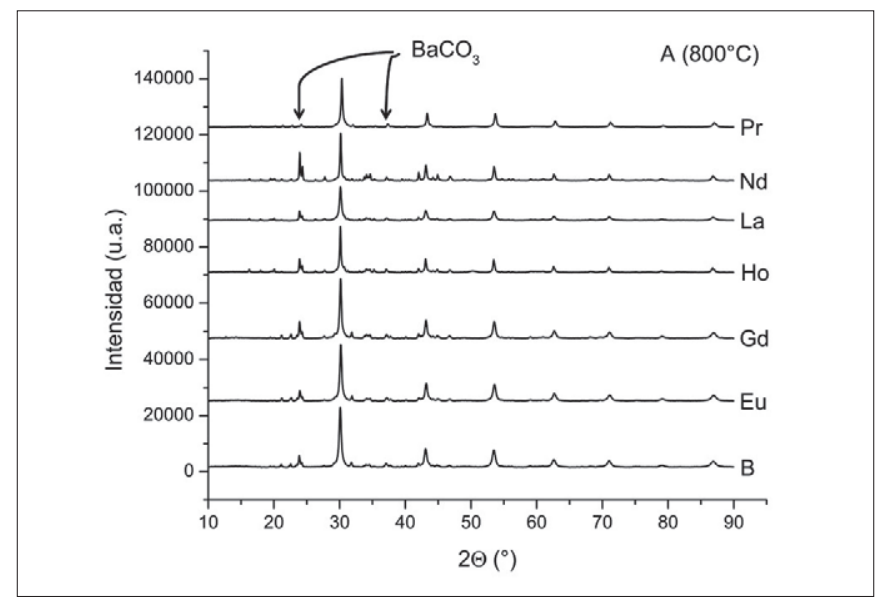

Figura 1. a) Patrones de difracción de rayos $\mathrm{X}$ de circonato de bario dopado con lantánidos a $800^{\circ} \mathrm{C}$.

una tendencia entre el tamaño de la partícula y el radio del lantánido dopante usado, sin embargo, se encuentra para el sólido dopado con europio el mayor tamaño.

El efecto de la temperatura sobre las fases obtenidas se manifiesta con la disminución de fases secundarias como el carbonato de bario. Al calcinar los sólidos a $900{ }^{\circ} \mathrm{C}$ por 30 minutos, Figura 1 b), la intensidad de las señales correspondientes a las fases secundarias disminuye de manera significativa para desaparecer por completo cuando la temperatura de calcinación se lleva a los $1000^{\circ}$ (Figura 1 c)) durante 30 minutos. En los difractogramas no se evidencian señales de intensidad significativa correspondientes a la presencia de los lantánidos trivalentes usados como dopantes; esto tiene su explicación en los límites de sensibilidad del método y el bajo nivel de dopaje usado en esta investigación. 


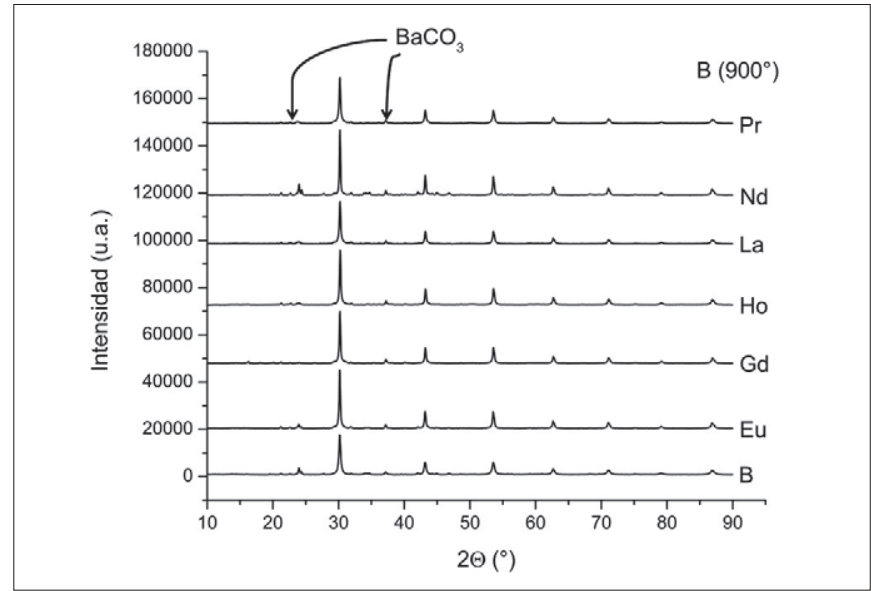

Figura 1. b) Patrones de difracción de rayos $\mathrm{X}$ de circonato de bario dopado con lantánidos a $900{ }^{\circ} \mathrm{C}$.

La literatura reporta resultados similares en el patrón de difracción de rayos X para circonato de bario preparado por distintos métodos. Es así como Prastomo(12), quien utilizó para la síntesis un método sol gel modificado, encuentra pequeñas cantidades de carbonato de bario y de óxido de circonio tetragonal que se eliminan cuando realiza la calcinación a los $1000{ }^{\circ} \mathrm{C}$. Boschini et al. ${ }^{14}$ prepararon el circonato de bario por un método solvotérmico (precipitación alcalina a bajas temperaturas) y reportaron la misma distribución de señales en el patrón de difracción de rayos X; además, encontraron una pequeña fase de carbonato de bario cuya presencia cobra importancia con el tiempo de almacenamiento del producto. Pfaff ${ }^{15}$, preparó circonato de bario por el método peroxo oxalato, a partir de $\mathrm{ZrOCl}_{2}$ y $\mathrm{BaCl}_{2}$ y obtuvo, luego de calcinar a $900{ }^{\circ} \mathrm{C}$ ( $1 \mathrm{~h}$ en atmósfera de aire), patrones de difracción en los cuales es evidente la presencia de $\mathrm{BaCO}_{3}$. Parida et al. (15) preparó el circonato de bario por el método cerámico convencional y lo sinterizó a $1670{ }^{\circ} \mathrm{C}$ por 4 horas; el compuesto obtenido indexó con el patrón ICDS No 970406, indicando una estructura cúbica. Yamanaka et al. (16) reportó similares resultados cuando usó el método cerámico convencional y calcinó a $1550{ }^{\circ} \mathrm{C}$. BorjaUrby et al. (17) usando un método hidrotérmico preparon el circonato de bario y lo indexaron con el patrón JCPDS 030699. Similares resultados han sido reportados por Cavalcante et al. (18), Sin et al. (19), Dias y Ciminelli (20), Leonard et al. (21), Glaucia et al. (22) y Thongtha et al. (23).

Atendiendo a las ideas antes expuestas, el circonato de bario obtenido por el método propuesto, citrato amorfo; muestra las mismas características del obtenido por los diversos métodos consultados y mencionados antes. El procedimiento propuesto presenta ventajas comparativas con los demás métodos en función a su sencillez y escasos requerimientos técnicos por lo que se espera que se reduzcan los tiempos de síntesis, las etapas intermedias propias de los otros métodos de síntesis y de la misma forma sea más económica su producción.

\subsection{Caracterización eléctrica}

\subsubsection{DIAGRAMAS TIPO BODE}

El diagrama de Bode (Figura 2) para el circonato de bario libre de dopantes, usado como referencia en esta investigación, muestra grandes variaciones en la impedancia, los valores registrados oscilan entre $10^{4}$ y $10^{8} \Omega$, la tendencia observada es la disminución

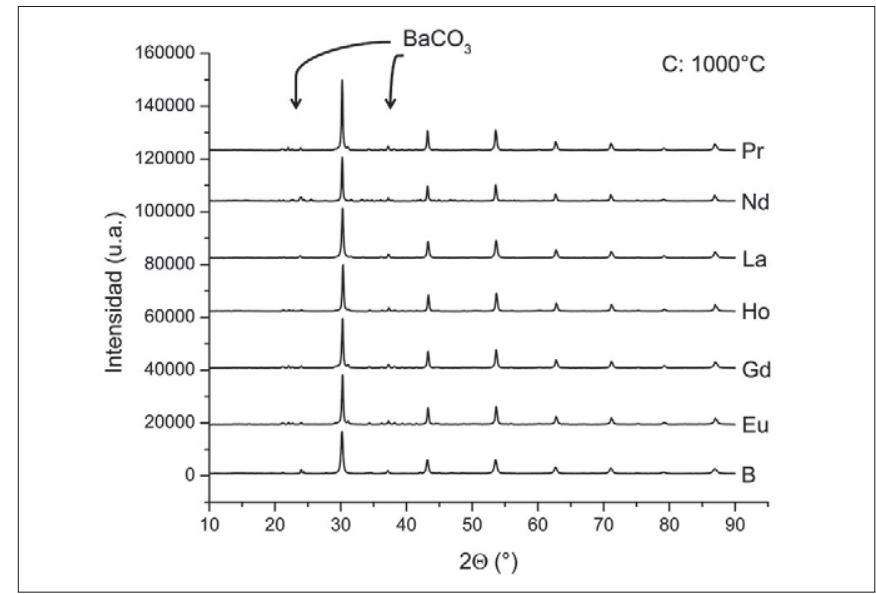

Figura 1. c) Patrones de difracción de rayos $\mathrm{X}$ de circonato de bario dopado con lantánidos a $1000{ }^{\circ} \mathrm{C}$.

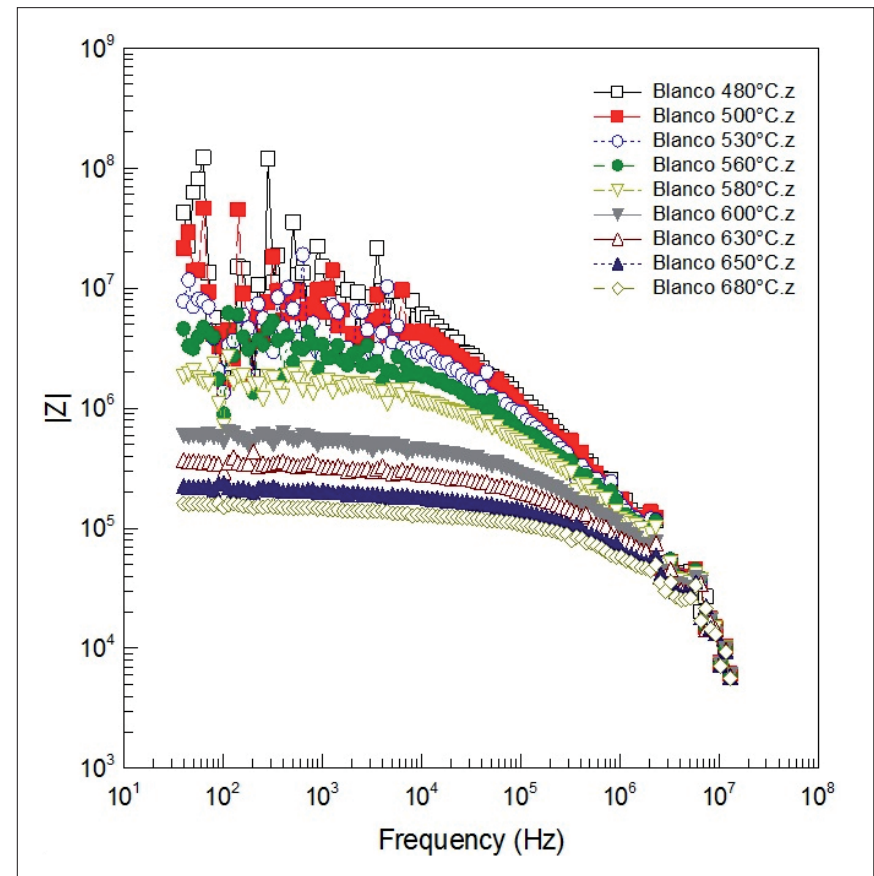

Figura 2. Diagramas de Bode para el circonato de bario.

de la impedancia frente al incremento de la frecuencia; con lo que la mayor variabilidad en las medidas se presenta en las mediciones tomadas a bajas frecuencias. $\mathrm{Al}$ apreciar el efecto que la variación de la temperatura ejerce sobre la impedancia de la muestra, se vislumbran dos tipos de comportamiento; el primero de ellos consiste en la alta variabilidad en las mediciones de impedancia hechas en el intervalo comprendido entre los 480 y los $580^{\circ} \mathrm{C}$ que es más notoria a bajas frecuencias; el segundo tipo de comportamiento consiste en la disminución de la impedancia ante el incremento en la temperatura, comportamiento que se ajusta al exhibido por los semiconductores que disminuyen su resistencia cuando se incrementa la temperatura. La disminución de la resistencia es bastante significativa, de aproximadamente tres órdenes de magnitud cuando se opera a bajas frecuencias y más bien con tendencia a llegar a un valor constante cuando se alcanzan frecuencias altas.

La variabilidad en las medidas obtenidas en la muestra de referencia a temperaturas inferiores a los $600^{\circ}$ no permitió 

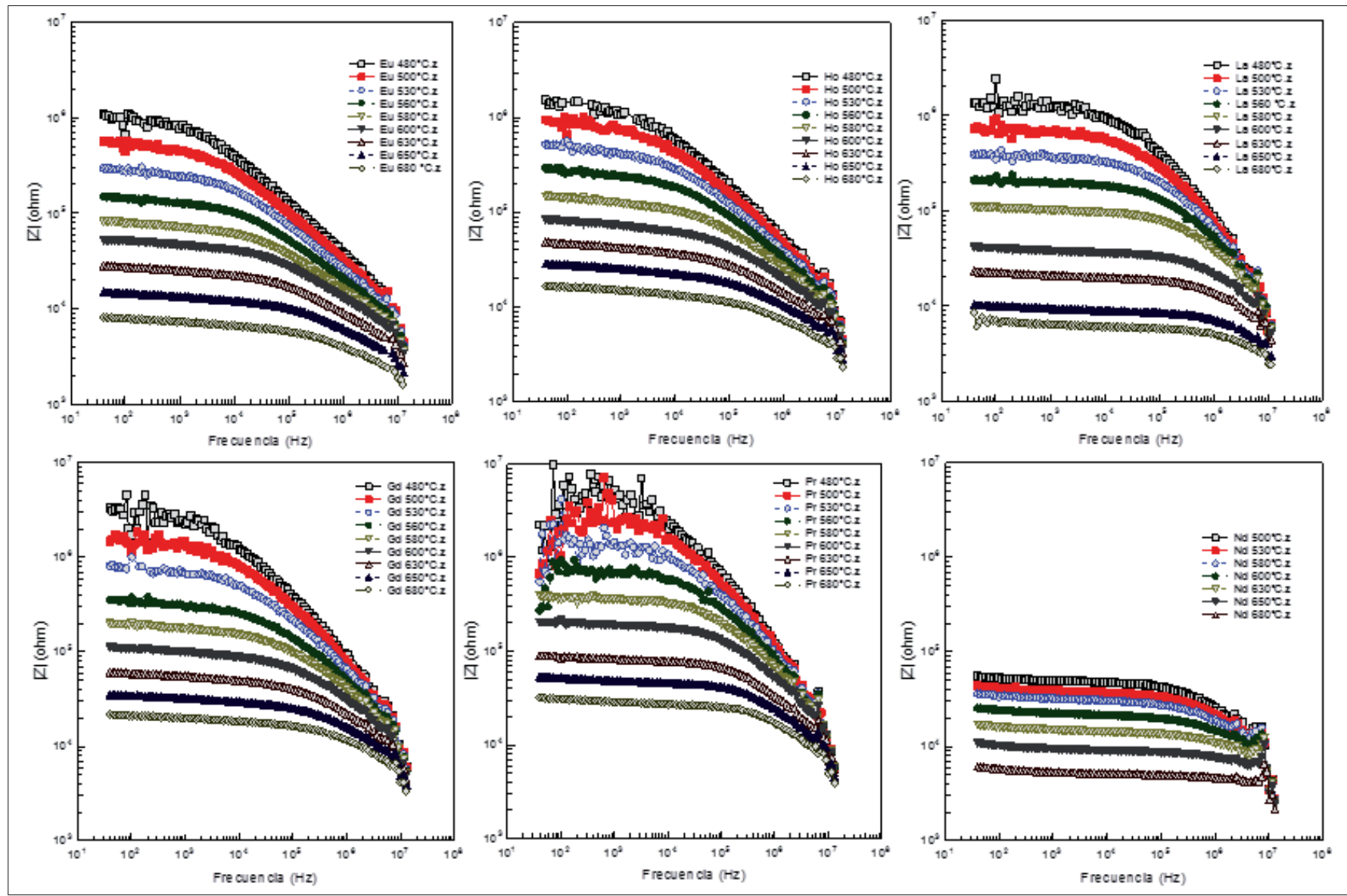

Figura 3. Diagramas de Bode para circonato de bario dopado.

tenerlas en cuenta, se analizaron por ello las mediciones comprendidas en el intervalo de los 600 a $680^{\circ} \mathrm{C}$. En este intervalo, a bajas frecuencias se presentan variaciones en la impedancia que no superan un orden de magnitud. De otra parte, al examinar los valores medidos a una misma temperatura, las variaciones encontradas se aproximan a tres órdenes de magnitud cuando la frecuencia se incrementa.

Para los sólidos dopados con lantánidos (Figura 3), las respuestas obtenidas permiten establecer tres tipos de comportamiento, el primero de ellos está corresponde a los sólidos dopados con europio, holmio y lantano. Estos muestran un patrón de conducta semejante, en líneas generales, al seguido por el material de referencia, las variaciones de la impedancia a bajas frecuencias oscilan entre $10^{4}$ y $10^{6} \Omega$ en el intervalo de temperaturas estudiado (de 480 a $680^{\circ} \mathrm{C}$ ), así mismo se encuentra la tendencia hacia un valor común independiente de la temperatura cuando se realiza el análisis en el dominio de las altas frecuencias.

Un segundo patrón de conducta es el mostrado por los materiales dopados con praseodimio y gadolinio; para éstos se encuentra variaciones grandes de la impedancia a bajas temperaturas, esta variabilidad disminuye a partir de los $560{ }^{\circ} \mathrm{C}$ para el circonato de bario dopado con praseodimio y de los $530^{\circ} \mathrm{C}$ para el dopado con gadolinio.

El tercer tipo de comportamiento lo presenta circonato de bario dopado con neodimio, su patrón de conducta es muy disímil con respecto a los otros sólidos examinados en este estudio, las mediciones de impedancia muestran una menor variabilidad que los sólidos anteriores y permanece

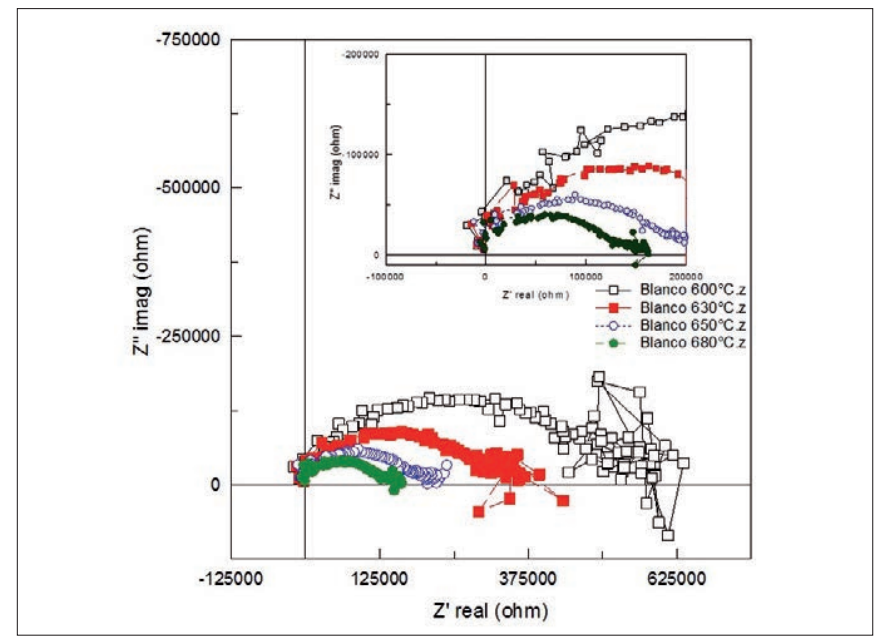

Figura 4. Diagrama de Nyquist para el circonato de bario.

casi constante en un amplio intervalo de frecuencias, tan solo disminuye levemente a partir de $10^{5} \mathrm{~Hz}$, además de lo anterior, es necesario anotar que el incremento en la temperatura no muestra el marcado efecto en la impedancia a bajas frecuencias que muestran los otros sólidos sometidos a estudio.

\subsubsection{DIAGRAMAS DE NYQUIST}

El diagrama de plano complejo para el circonato de bario sin dopar tomado a temperaturas entre 600 y $680^{\circ} \mathrm{C}$ se presenta en la Figura 4. Las mediciones realizadas a temperaturas 

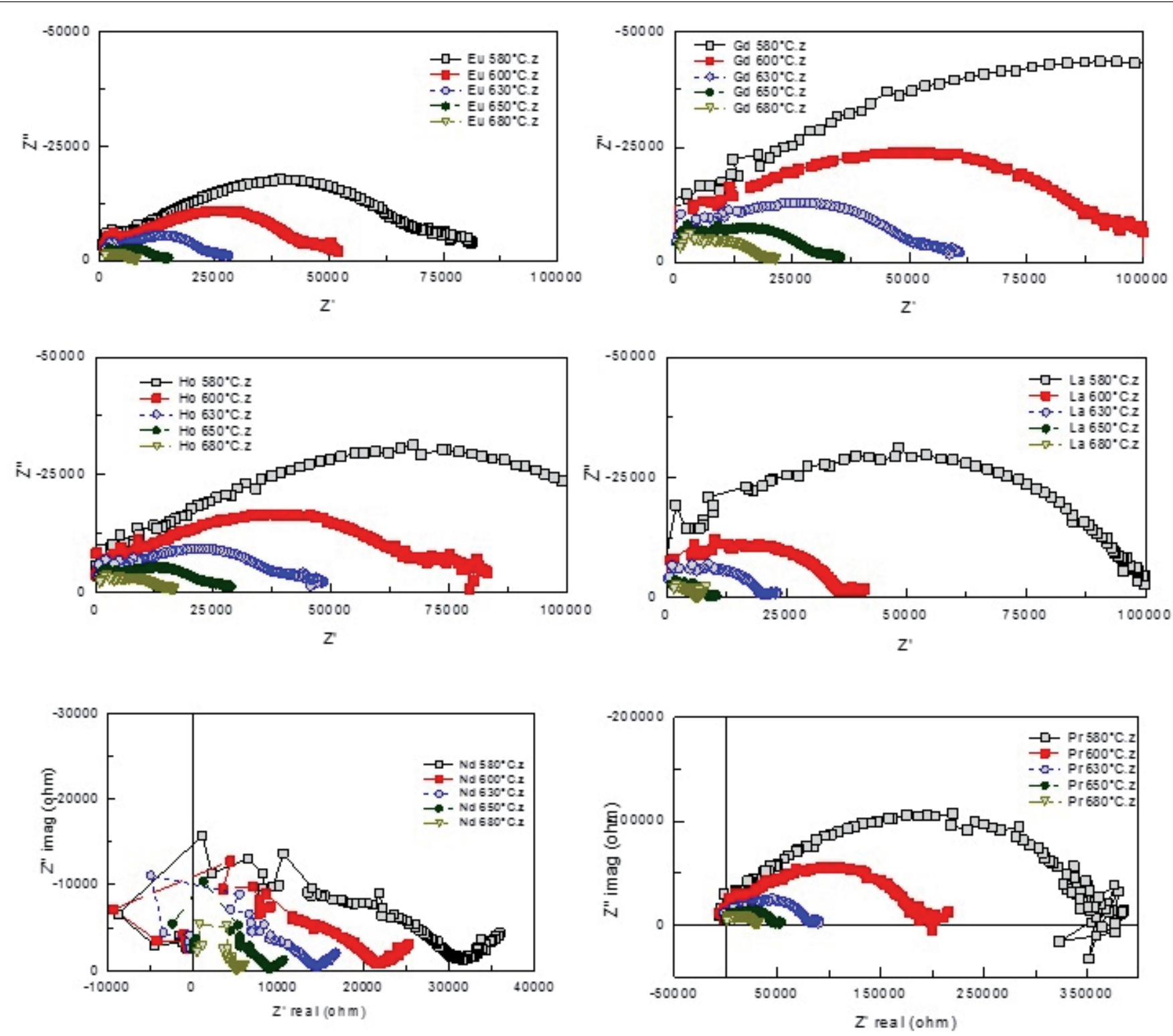

Figura 5. Diagrama de Nyquist para los sólidos dopados.

inferiores a $\operatorname{los} 600^{\circ} \mathrm{C}$ muestran tal variabilidad que no permitió considerarlas en este análisis. Esta variabilidad se hace muy evidente a frecuencias bajas, el inserto de la Figura 4 permite apreciar la mejora en la señal que se presenta con el incremento de la temperatura. A $680^{\circ} \mathrm{C}$ el gráfico muestra un único semicírculo seguido de una zona plana, por lo anterior, este diagrama no permite la diferenciación de la conducción debida al volumen del grano de la que se presentan en el límite de éste, aun incrementando la temperatura hasta los $680^{\circ} \mathrm{C}$.

Para los sólidos dopados con los lantánidos, en la Figura 5 se muestran los diagramas de Nyquist a temperaturas entre 580 y $680{ }^{\circ} \mathrm{C}$. El incremento en la temperatura de análisis conduce a la disminución de la impedancia, que como se había mencionado antes es un comportamiento presentado de manera regular por los semiconductores. La variabilidad en las medidas, mencionada antes, se manifiesta a bajas temperaturas y es muy evidente en los diagramas correspondientes al circonato de bario dopado con gadolinio, lantano y praseodimio. Para los sólidos dopados con holmio y europio se aprecian respuestas mucho más homogéneas, se presenta un semicírculo en el que no es posible, en estas condiciones de análisis diferenciar las respuestas del volumen y del límite de grano. Caso especial es el comportamiento mostrado por el circonato de bario dopado con neodimio; su respuesta en el dominio de las altas frecuencias reporta impedancias negativas con independencia de la temperatura a la cual se verifica el análisis, además, se presenta una muy alta variabilidad en este dominio. El comportamiento de este material es atípico en relación al exhibido por los otros sólidos aquí estudiados y se repite lo observado en los diagramas de Bode en los que la respuesta dada por el sólido no concuerda con las tendencias generales mostradas tanto por el circonato de bario usado como blanco como por los materiales dopados.

\subsubsection{CIRCUITOS EQUIVALENTES}

Los diagramas de Nyquist obtenidos muestran solapadas las señales características del volumen de grano y del límite del mismo, debido a factores como los tiempos de relajación, 


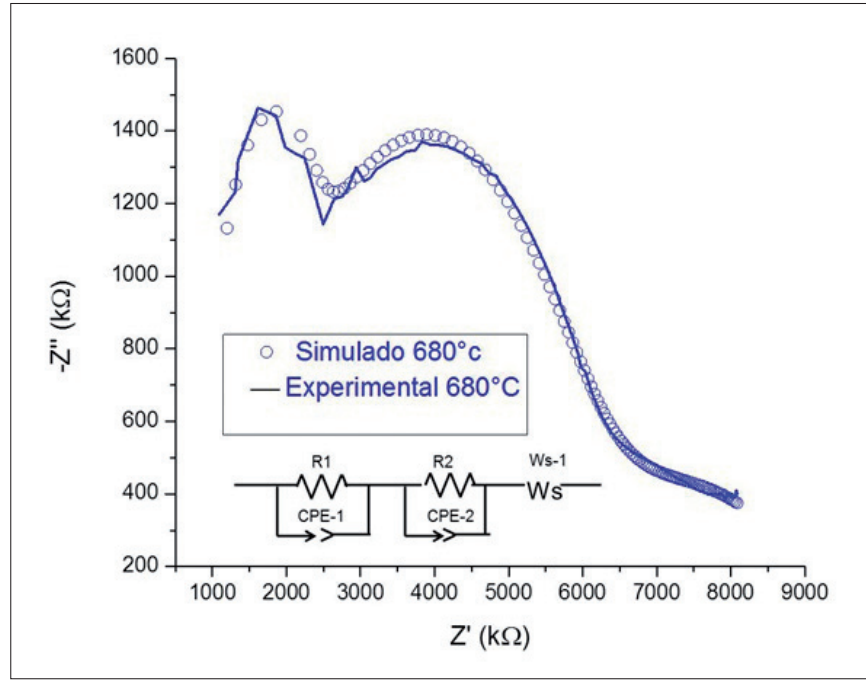

Figura 6. Diagramas de Nyquist, simulado y real para circonato de bario dopado con europio a $680{ }^{\circ} \mathrm{C}$.

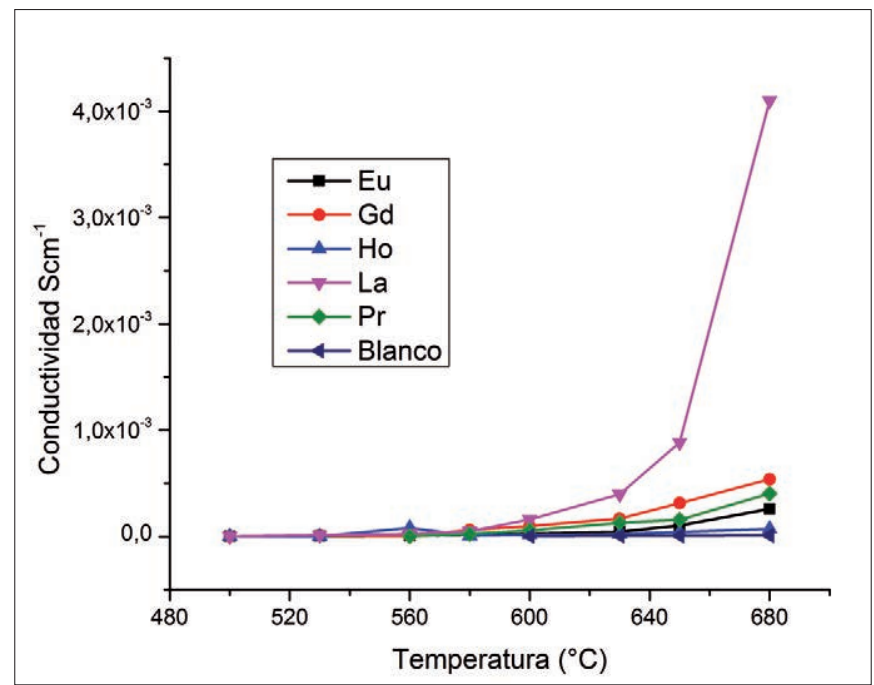

Figura 8a. Conductividad eléctrica debida al volumen de grano para circonato de bario sin dopar y dopado con lantánidos trivalentes.

la orientación y distribución de los granos del sólido fue posible proponer un modelo de circuito equivalente que permite una interpretación del comportamiento de las curvas experimentales obtenidas, con la ayuda de Zview ${ }^{\circledR}$. Para el caso particular del sistema $\mathrm{BaZrO}_{3}$, dopado con $0,03 \%$ de europio, estudiado a 680 C, el circuito equivalente se ilustra en la Figura 6.

El circuito está conformado por tres componentes, dos circuitos RC en paralelo ubicados en serie y un elemento tipo Warburg; el primero y segundo circuitos representan el comportamiento del volumen del grano (bulk) y de los límites del grano respectivamente; están conformados por una resistencia y un elemento de fase común, conectados en paralelo; el elemento de fase común por lo regular representa un comportamiento no ideal de la capacitancia del sistema, esta desviación del comportamiento ideal obedece a las interacciones que se pueden presentar entre el sólido estudiado y el electrodo, es decir a posibles interacciones químicas, así como a la rugosidad de la superficie. Por último el elemento tipo Warburg por lo general está asociado a fenómenos de difusión que se presentan a frecuencias altas.

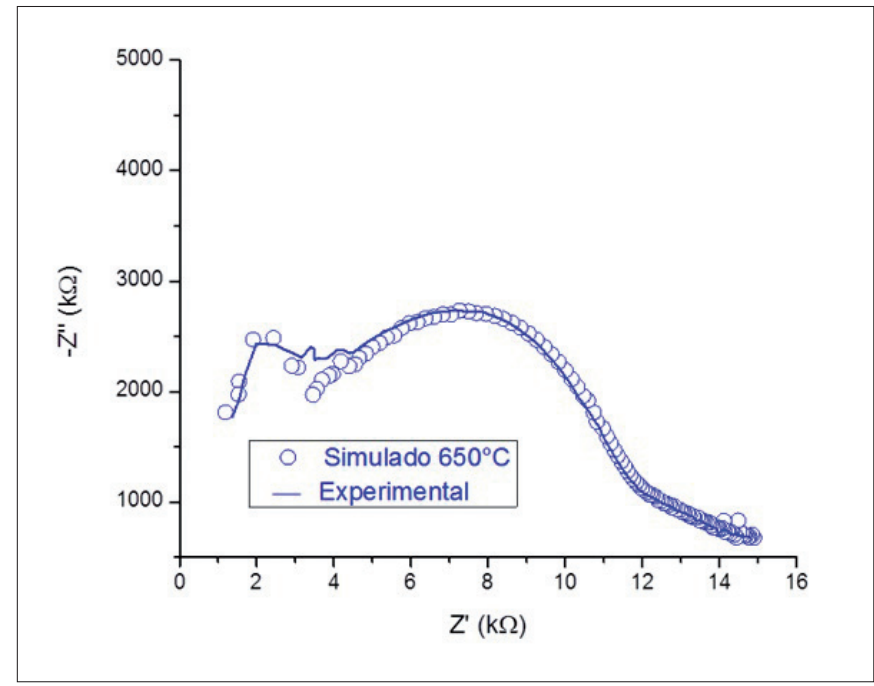

Figura 7. Diagramas de Nyquist, simulado y real para circonato de bario dopado con europio a $650{ }^{\circ} \mathrm{C}$.

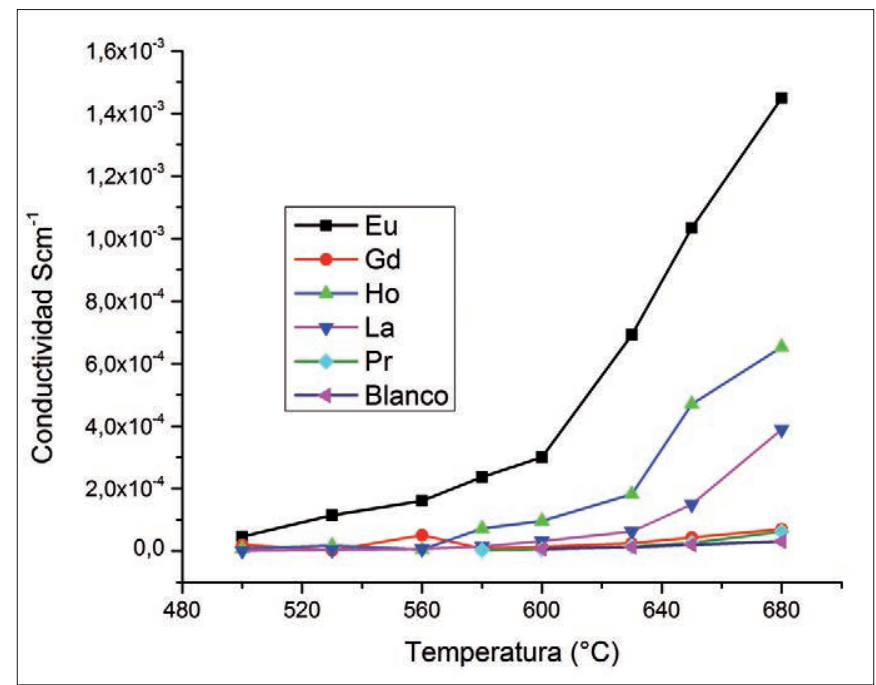

Figura 8b. Conductividad eléctrica debida al límite del grano para circonato de bario sin dopar y dopado con lantánidos trivalentes.

En la Figura 6 también se aprecia el diagrama de Nyquist para el sólido dopado con europio tomado a $680^{\circ} \mathrm{C}$, junto a éste se muestra la simulación del circuito equivalente; el ajuste es bueno con algunas limitaciones en el dominio de las altas frecuencias, este fenómeno se observa mejor en la Figura 7, que corresponde al comportamiento observado a $650{ }^{\circ} \mathrm{C}$. Las desviaciones obtenidas para $Z^{\prime}$ comprendidos entre 3 y $5 \mathrm{k} \Omega$, tienen su origen en la alta dispersión que muestran los datos experimentales.

\subsubsection{CONDUCTIVIDAD}

Usando la información correspondiente a los circuitos equivalentes asignados a los sólidos sintetizados se determinaron las resistencias del volumen del grano y la del límite del mismo, con éstos se calcularon las conductividades de cada material en las temperaturas estudiadas. Las gráficas del tipo Arrhenius mostradas en las Figuras 8a y 8b, las cuales son útiles para el cálculo de las energías de activación (Tabla 2), permiten establecer dos tipos de conducta en la conductividad debida al volumen del grano, los sólidos dopados con gadolinio 


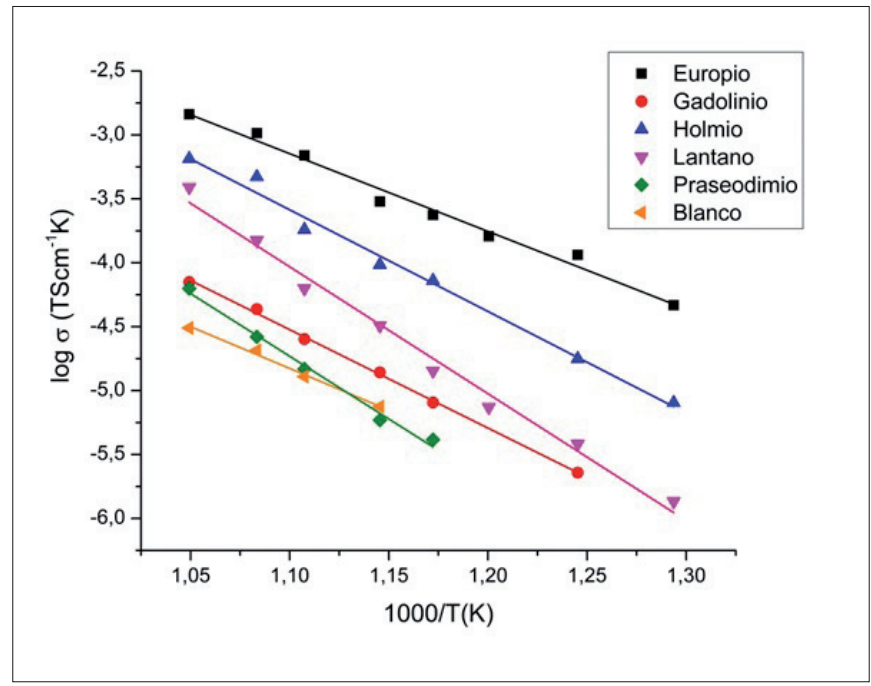

Figura 9a. Curvas de Arrhenius para circonato de bario sin dopar y dopado con lantánidos trivalentes. Aporte del volumen del grano.

y con praseodimio presentan conductividades que no difieren entre sí, desde esta perspectiva, la adición de estos cationes no ocasiona mejoras en la conductividad del circonato de bario; de otra parte para los sólidos dopados con lantano, holmio y europio se aprecian mejoras en la conductividad de hasta dos órdenes de magnitud siendo más drásticos los cambios en el circonato de bario dopado con europio. Se advierte además que a bajas temperaturas las variaciones en la conductividad son menos notorias que las observadas a temperaturas superiores a $600{ }^{\circ} \mathrm{C}$.

Las conductividades debidas al límite de grano, tan solo presentan ligeras variaciones a excepción del sólido dopado con lantano, en este material la conductividad debida al borde del grano muestra un incremento muy marcado, en relación a los otros sólidos estudiados. Estas variaciones en la conductividad son de alrededor de dos órdenes de magnitud superiores a las encontradas para el borde del grano, excepto para el circonato de bario dopado con lantano.

A partir de un ajuste por regresión lineal se calculó la energía de activación tanto de la conductividad en el volumen del grano como en el límite de grano, los resultados se presentan en la Tabla 2. Los valores de energía de activación son muy cercanos entre sí y próximos a los $0.55 \mathrm{eV}$, energía correspondiente a la conducción de protones como lo mencionan Fabbri et al(24) quienes examinaron el circonato de bario como material para cátodos de celdas de combustible de temperatura intermedia sintetizado por un método de combustión.

\section{CONCLUSIONES}

El método de síntesis propuesto permite obtener circonato de bario en condiciones más favorables que las exhibidas por el método convencional. Es posible reducir los costos de síntesis de circonato de bario usando este método, la reducción se evidencia en el calentamiento a menores temperaturas, por periodos más cortos y sin el requerimiento de someter los precursores a presiones elevadas.

El calentamiento a $800{ }^{\circ} \mathrm{C}$ por una hora permite obtener el circonato de bario con grupo espacial $\mathrm{Pm}-3 m(221), \mathrm{Z}=1$,

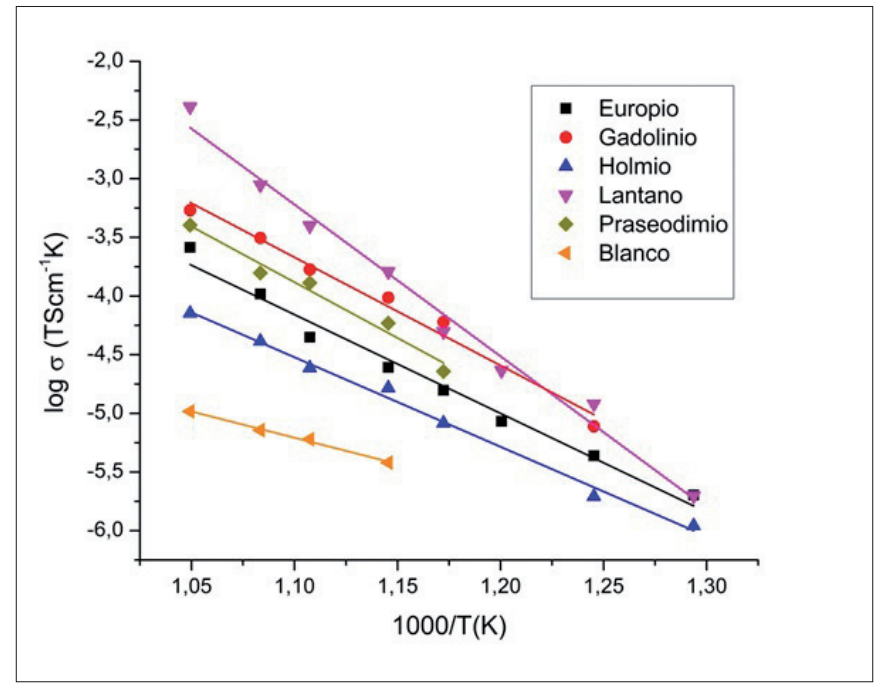

Figura 9b. Curvas de Arrhenius para circonato de bario sin dopar y dopado con lantánidos trivalentes. Aporte del límite del grano.

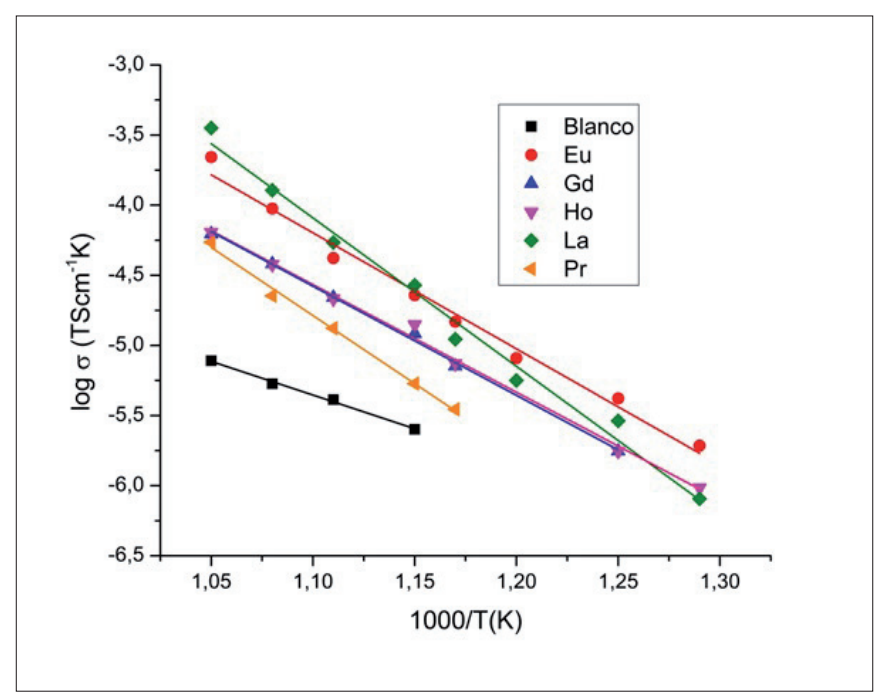

Figura 9c. Curvas de Arrhenius para circonato de bario sin dopar y dopado con lantánidos trivalentes. Conductividad total.

sistema cristalino cúbico con parámetro de celda $a=4,193 \AA ̊$ y volumen de celda de 73,718 $\AA^{3}$. A esta temperatura se presentan cantidades remanentes de carbonato de bario y de óxido de circonio que requieren tratamiento a mayor temperatura para su eliminación completa.

Este método de síntesis produce, además, materiales nanoestructurados con tamaños de cristalitomenores para todos los casos a $50 \mathrm{~nm}$, tamaño que se pueden considerar óptimo para las aplicaciones en el campo de las electrocerámicas.

La inclusión de lantánidos trivalentes como dopantes no presenta cambios significativos en la estructura cristalina del circonato de bario al ser analizado por difracción de rayos $\mathrm{X}$.

La variabilidad en las medidas de impedancia disminuye con la frecuencia y la temperatura, en particular los mejores resultados se encuentran a temperaturas superiores a los $580^{\circ} \mathrm{C}$.

Lossólidos dopadosconlantano, holmioyeuropiopresentan mejorías en la conductividad eléctrica en comparación con el circonato de bario usado como referencia. 


\section{AGRADECIMIENTOS}

Este proyecto pudo llevarse a cabo gracias a la financiación de la Universidad Nacional de Colombia y la Universitat Jaume I de Castellón de la Plana, Comunidad Valenciana, España.

\section{REFERENCIAS}

1. Wincewicz, K., and Cooper, J. (2005) Taxonomies of SOFC material and manufacturing alternatives, J. Power Sources 140, 280-296.

2. Moon, H., Kim, S. D., Hyun, S. H., and Kim, H. S. (2008) Development of IT-SOFC unit cells with anode-supported thin electrolytes via tape casting and co-firing, Int. J. Hydrogen Energy 33, 1758-1768.

3. Hui, S. (Rob), Roller, J., Yick, S., Zhang, X., Decès-Petit, C., Xie, Y., Maric, R., and Ghosh, D. (2007) A brief review of the ionic conductivity enhancement for selected oxide electrolytes, J. Power Sources 172, 493-502.

4. Haile, S. M. (2003) Materials for fuel cells, Mater. Today 6, 24-29.

5. Sammells, A., Cook, R., and White, J. H. (1992) Rational selection of advanced solid electrolytes for intermediate temperature fuel cells, Solid State Ionics 52, 111-123.

6. Iwahara, H. (1988) High temperature proton conducting oxides and their applications to solid electrolyte fuel cells and steam electrolyzer for hydrogen production, Solid State Ionics 28, 573-578.

7. Yajima, T., and Iwahara, H. (1991) CaZrO3-type hydrogen and steam sensors: trial fabrication and their characteristics, Sensors Actuators B 5, 145-147.

8. Yajima, T., Kazeoka, H., Yogo, T., and Iwahara, H. (1991) Proton conduction in sintered oxides based on CaZrO3, Solid State Ionics 47, 271-275.

9. Kreuer, K. D. (2003) Proton-Conducting Oxides, Annu. Rev. Mater. Res. 33 333-359.

10. Winter, M., and Brodd, R. J. (2004) What Are Batteries, Fuel Cells, and Supercapacitors?, Chem. Rev. 104, 4245-4270.

11. Bhagwat, M. (2004) Synthesis of nanocrystalline zirconia by amorphous citrate route: structural and thermal (HTXRD) studies, Mater. Res. Bull. 39, 1627-1640.

12. Prastomo, N., Zakaria, N. H. B., Kawamura, G., Muto, H., Sakai, M., and Matsuda, A. (2011) High surface area BaZrO3 photocatalyst prepared by base-hot-water treatment, J. Eur. Ceram. Soc. 31, 2699-2705.

13. Moreira, L., and Andre, J. (2009) Synthesis of Fine Micro-sized BaZrO3 Powders Based on a Decaoctahedron Shape by the Microwave-Assisted Hydrothermal Method, Cryst. Growth Des. 9, 833-839.
14. Moreira, M. L., Volanti, D. P., Andrés, J., Montes, P. J. R., Valerio, M. E. G., Varela, J. a., and Longo, E. (2011) Radioluminescence properties of decaoctahedral BaZrO3, Scr. Mater. 64, 118-121.

15. Parida, S., Rout, S. K., Cavalcante, L. S., Sinha, E., Li, M. S. Subramanian, V., Gupta, N., Gupta, V. R., Varela, J. a., and Longo, E. (2012) Structural refinement, optical and microwave dielectric properties of BaZrO3, Ceram. Int., Elsevier Ltd and Techna Group S.r.l. 38, 2129-2138.

16. Yamanaka, S., Hamaguchi, T., Oyama, T., Matsuda, T., Kobayashi, S., and Kurosaki, K. (2003) Heat capacities and thermal conductivities of perovskite type $\mathrm{BaZrO3}$ and $\mathrm{BaCeO} 3$, J. Alloys Compd. 359, 1-4.

17. Borja-Urby, R., Diaz-Torres, L. a., Salas, P., Angeles-Chavez, C., and Meza, O. (2011) Strong broad green UV-excited photoluminescence in rare earth $(\mathrm{RE}=\mathrm{Ce}, \mathrm{Eu}, \mathrm{Dy}, \mathrm{Er}, \mathrm{Yb})$ doped barium zirconate, Mater. Sci. Eng. B, Elsevier B.V. 176, 1388-1392.

18. Cavalcante, L. S., Sczancoski, J. C., Espinosa, J. W. M., Mastelaro, V. R., Michalowicz, a., Pizani, P. S., De Vicente, F. S., Li, M. S., Varela, J. a., and Longo, E. (2009) Intense blue and green photoluminescence emissions at room temperature in barium zirconate powders, J. Alloys Compd. 471, 253-258.

19. Sin, A., Montaser, B., Odier, P., and Weiss, F. (2002) Synthesis and Sintering of Large Batches of Barium Zirconate Nanopowders, J. Am. Ceram. Soc. 85, 1928-1932.

20. Dias, A., and Ciminelli, V. S. T. (2003) Electroceramic Materials of Tailored Phase and Morphology by Hydrothermal Technology, Chem. Mater. 15, 1344-1352.

21. Leonard, K. J., Sathyamurthy, S., and Paranthaman, M. P. (2005) Characterization of BaZrO3 Nanoparticles Prepared by Reverse Micelle Synthesis, Chem. Mater. 17, 4010-4017.

22. Moreira, M. L., Buzolin, P., Longo, V. M., Nicoleti, N. H., Sambrano, J. R., Li, M. S., Varela, J., and Longo, E. (2011) Joint Experimental and Theoretical Analysis of Order-Disorder Effects in Cubic BaZrO3 Assembled Nanoparticles under Decaoctahedral Shape, J. Phys. Chem. A $115,4482-4490$.

23. Thongtha, A., and Bongkarn, T. (2009) Phase Formation and Microstructure of Barium Zirconate Ceramics Prepared Using the Combustion Technique, Ferroelectrics 383, 33-39.

24. Fabbri, E., Markus, I., Bi, L., Pergolesi, D., and Traversa, E. (2011) Tailoring mixed proton-electronic conductivity of $\mathrm{BaZrO} 3$ by $\mathrm{Y}$ and Pr co-doping for cathode application in protonic SOFCs, Solid State Ionics, Elsevier B.V. 202, 30-35.

Recibido:09/09/2013

Recibida versión corregida: 26/02/2014

Aceptado: 26/02/2014 